\title{
Quantitative Techniques and Its Role in Transport Management
}

\author{
Bhadmus Ola ${ }^{1}$, Oseni, B. Azeez ${ }^{2}$, Mabosanyinje Adedeji \\ 1Department of Geography and Environmental Management (Transport Unit), Tai Solarin University of \\ Education, Ijebu-Ode \\ 2 Department of Mathematics and Statistics. The Polytechnic, Ibadan \\ 3Department of Statistics and Mathematics, Moshood Abiola Polytechnic, Abeokuta.
}

\begin{abstract}
We focused attention on the Quantitative techniques and its significance roles in transport management. Emphasis is on the parametric statistics tests that are commonly used in transport management. The parametric statistics Z-test, $t$-test and F-test were fully discussed in this paper and its applications in transport management when testing for one sample means, two sample means and three or more sample means. The paper also reveals that parametric tests are more powerful than their non-parametric counterpart.
\end{abstract}

Finally, the condition(s) for using $t, z$ and $F$-test were fully discussed.

Keywords: Parametric, non-parametric, scaling ordinal, nominal, inductive, deductive, homoscedasticity

\section{Introduction}

The role of quantitative techniques in transport management can not be over emphasized. Quantitative techniques implies two important things namely (i) ability to measure or assign numbers to variables, attributes or whatever the characteristics of interest and (ii) ability to apply analytical technique of mathematics or statistics to the data of interest. As a matter of fact, quantitative techniques consist of inferences from the two main but related fields of mathematics and statistics. Mathematics is chiefly deductive in nature while statistics is primary inductive. In deductive argument, no additional information is required to reach a firm conclusion. Deductive reasoning does not offer explanation beyond the data.

On the other hand, inductive arguments provide conditions which in some sense exceed the content of the premises upon which they are based.

The word statistics can be broadly defined as a method of data collection and analysis of data in such a way as to minimized any uncertainty in the conclusion drawn from the data and be able to access the degree of such uncertainty. Vital statistics such as birth rates, economic indicators such as employment rates and money supply figures and social statistics such as juvenile delinquency rate are all statistics used in this sense of the word.

The word statistics has another specialized meaning. It is the methodology for collecting; presenting can be used as a basis for investigation in such diverse academic fields such as education, physics, engineering, medicine, biological sciences, and social sciences including transport.

Finally, quantitative techniques in transport management is designed to acquaint the students with a variety of analytical methods currently in use in transport studies.

The following are some of the role of quantitative techniques:

i. Statistics plays a vital role in transport planning and management in testing of hypothesis and decision making. This is more so because transport as a disciplines are regarded as science and so must follow the culture of science, one of whose requirements is the vigorous testing of hypothesis. In the physical sciences like physics and chemistry, the frame work for achieving such vigour is provided by the laboratory, but it is statistics that does the same for the social sciences as whole.

ii. Also, in these disciplines, it is not often possible to obtain data from the whole population of interest, sample are then taken to generalize for the entire population of interest. This is done via inferential statistics that will leads to estimation of parameters of the population and testing of hypothesis.

iii. Statistics provides us with the methods by which the samples themselves may be taken in order that they may be representative of the population data through sampling techniques.

iv. Statistical techniques provide the tools in forecasting, projections and predictions in knowing the future demand of services such as transport, hospital, educational services and several other phenomena in which the disciplines are interested.

v. In transport planning and social sciences generally, we deal with uncertainties. These uncertainties about our data are proned to substantial degrees of errors. These are also uncertainties about the predictability of human behaviour which forms a considerable part of our interests. These uncertainties are better studied by utilizing the statistical technique of probability. 
vi. Quantitative methods expose the student to those new techniques from statistics and mathematics that have revolutionized the way researches are done in various disciplines including transport study which is our interest of this paper.

\section{Sources of Data in Transport Management Studies}

There are two main sources of data in the social and management sciences generally. These are primary data such as interview, census, direct observation methods, sample surveys, recording of transactions, questionnaire, observation and experimental method and secondary data like information obtainable from government publications, books, consultancy reports, data banks, journals, conferences paper and even newspapers.

\subsection{Methods of Measurement}

In social and management sciences generally information is obtained through direct measurement in the field. Such information like measure distances, speeds, income, expenditure, mass, volume, weight, height etc. It is necessary to introduce the reader to various measurements. This is so, because the type of measurement determines the type of statistical techniques that can be used to analyse the data.

The following are the types of scale in social sciences namely: Nominal scaling-it involves numbering of objects or events and these numberings act no more than as names/identification; ordinal scaling-this is where we order or rank events or objects from the highest to the smallest or from most to the least, that is in order of magnitude; interval scaling-we are able to specify how far apart the magnitudes are from each other, that is we can measure the distance between two objects on the scale which is what we could not do with ordinal scale; ratio scaling-this is one in which data possess a natural origin; multidimensional scaling-to study phenomenon that cannot be reasonably measured by one variable.

Moreover, the reader should note that parametric statistics can be applied to interval and ratio scale while non-parametric statistics can be applied to nominal and ordinal scale.

\subsection{Parametric Methods of Statistical Hypothesis Testing:}

The word parameter in statistics simply means the quantitative characteristics of the population rather than the sample. Parametric statistics are those statistics that require that the population from which sample data is drawn must possess certain characteristics or conditions before they can be used. If all these conditions are met, parametric tests are more powerful than their non-parametric counterparts. In real practical applications, we do test whether these conditions are satisfied. In most cases we either unable, or do not bother to test for these conditions. Instead, we simply assume that the conditions have been met. The following are the conditions for parametric tests viz:

i. that the sample data are drawn from normally distributed populations.

ii. that the observations are independent i.e the selection of one observation from the population does not bias the chances of others i.e the observations are selected randomly.

iii. that the population of interest must have the same variance (homoscedasticity).

iv. that the variables must be measured on the interval or ratio scales so that arithmetic operations like addition, subtraction and division can be carried out.

In this paper, we shall discuss parametric tests which include the student's $t$-test and F-test statistic.

\subsection{1: Some Parametric Statistics test that commonly used in transport.}

Student's t-test: In transport management we are interested in testing hypothesis concerning the difference between a sample mean and a specified value which could be an assumed standard or theoretical; testing the hypothesis about the differences between two sample means. Student's t-test would be used if the number of samples is less that 30 and distribution- $Z$ if the number of samples involved is equal to or greater than 30 . The two test-statistics mentioned above are defined as

$$
Z=\frac{\bar{X}-\mu}{\sigma / \sqrt{n}}
$$

where $\bar{X}$ =sample mean, $\mu$ = population mean, $\sigma=$ population standard deviation and $\mathrm{n}=$ sample size. It should be noted here that, in most practical applications, the standard deviation of the population $(\sigma)$ is unknown and we have to replace it with the sample standard deviation(s). we then have $Z=\frac{\bar{X}-\mu}{s / \sqrt{n}}$ for one sample mean and

(ii) for two sample means

$$
Z=\frac{\bar{X}_{1}-\bar{X}_{2}}{\sqrt{\frac{S_{1}{ }^{2}}{n_{1}}+\frac{S_{2}{ }^{2}}{n_{2}}}}
$$




\section{Illustrations:}

\section{Case 1: One sample means.}

In Nigeria, the expiring period for national Driving Licence is 2 years. If a random sample of 50 drivers in a city is taken and the average time of their licences is 2.26years and a sample standard deviation of 0.66. Can we say that the drivers in that city violated the National regulation in driving licence?

Here, the problem is to determine whether or not the sample mean $(\bar{X})$ could have come from the same population with the mean of 2 years i.e the Null hypothesis $\mathrm{H}_{0}: \mu=2$ years against three possible alternatives hypothesis $\mathrm{H}_{1}$ are:

$\mathrm{H}_{1}: \mu \neq 2$ years (two-tailed test)

$\mathrm{H}_{1}: \mu>2$ years (one-tailed test)

$\mathrm{H}_{1}: \mu<2$ years (one-tailed test)

For any of these three hypothesis, we use the normal distribution

$$
Z=\frac{\bar{X}-\mu}{\sigma / \sqrt{n}}=\frac{2.26-2.0}{0.66 / \sqrt{50}}=2.79 \text { years }
$$

But $\mathrm{Z}(5 \%)=1.96$. since $\mathrm{Z}(5 \%)$ tabulated value is less than $\mathrm{Z}$ calculated $(2.79)$, the hypothesis $\mathrm{H}_{\mathrm{o}}$ is rejected in favour of $\mathrm{H}_{1}$ and conclude that the National regulation has actually been violated as the license had exceeded the stipulated time of 2 years.

\section{Case 2: Two sample means.}

A sample of cargo throughput in two seaports

$\mathrm{A}$ and $\mathrm{B}$ showed the following quantity in tones per month.

Port A (tones in thousand):3.5, 4.5, 2.0, 6.0, 6.5, 4.8,3.8, 7.5, 8.0 and 7.2.

Port B (tones in thousand): 4.5, 4.0, 4.8, 5.2, 3.2, 3.8, 7.8, 7.2, 6.1 and 3.4. The problem here is to investigate whether the cargo throughput differs significantly as between the two ports.

The two hypothesis can be posed thus:

$\mathrm{H}_{\mathrm{o}}: \mu_{\mathrm{A}}=\mu_{\mathrm{B}}$ versus $\mathrm{H}_{1}: \mu_{\mathrm{A}}>\mu_{\mathrm{B}}$

The mean of port $\mathrm{A}$ and port $\mathrm{B}$ are 5.38 and 5.0 respectively and their standard deviations are 1.97 and 1.56 respectively.

$$
\text { Hence, the test-statistic here is } t=\frac{5.38-5.0}{\sqrt{\frac{1.97^{2}}{10}+\frac{1.56^{2}}{10}}}=0.34 \text { using (ii) }
$$

But $\mathrm{t}_{18}(5 \%)=1.73$ from the table Hence, there is no significant difference in the cargo throughput of the two ports:

\section{Case3: Three or more sample means.}

Suppose we are interested in whether differences existed in the number of vehicles registered in three cities A, B and C. The following data were used to illustrates how three of more means can be analysed using F-

\begin{tabular}{|c|c|c|c|c|c|c|c|c|}
\hline City A: 80 & 70 & 70 & 50 & 90 & 100 & 50 & 95 & 40 \\
\hline City B: 80 & 60 & 70 & 60 & 50 & 60 & 65 & 75 & 35 \\
\hline City C: 80 & 75 & 45 & 35 & 65 & 60 & 25 & 35 & 25 \\
\hline
\end{tabular}
statistic via Analysis of variance table (ANOVA) developed by Fisher in 1947.

\section{The relevant hypothesis here is}

$\mathrm{H}_{\mathrm{o}}: \mu_{\mathrm{A}}=\mu_{\mathrm{B}}=\mu_{\mathrm{C}}$ against

$\mathrm{H}_{1}: \mu_{\mathrm{A}} \neq \mu_{\mathrm{B} .} \neq \mu_{\mathrm{C}}$ for some city

The analysis of variance table is

\begin{tabular}{|l|l|l|l|l|}
\hline Source of variation & Degree of freedom & Sum of square & Mean square & $\begin{array}{c}\text { F- } \\
\text { ratio }\end{array}$ \\
\hline Cities & 2 & 2105 & 1052.5 & 3.19 \\
\hline Error & 27 & 8912.5 & 330.0926 & - \\
\hline Total & 29 & 11017.5 & - & - \\
\hline
\end{tabular}

* F-critical: 3.35

It can be observed that there is no differences in the number of vehicles registered in three cities. 


\section{Conclusion}

We observed that the roles of quantitative techniques in transport industries and managed can not be neglected. This is because parametric statistics can be applied to interval and ratio scale while non-parametric statistics can be applied to nominal and ordinal scale.

\section{References:}

[1] Button, K. J. (1973): "Motor car ownership in the West Riding of Yorkshire. Some findings" Traffic Engineering and control.

[2] Boxton, M. J. and D.G. Rhys (1972): "The demand for car ownership: A note" Scottish Journal of Political Economy.

[3] Douglas, A.A (1973) "Home based trip end models-a comparison between category analysis and regression analysis procedures" Transportation, vol.2.

[4] Oluwasanmi, A. J. (1992): Road traffic trends in Nigeria. Acc. Anal Prev. 1993, 25,485-487.

[5] Asogwa, S.E, Obionu C. N. (1985): Urban Pedestrian accidents and the efficacy of a counter-measure. Centre. African J. medicine. 1985, 31: 62-65 